\title{
FORTALEZAS, CASTILLOS Y TORRES DE EXTREMADURA MEDIEVAL
}

\author{
POR \\ VALENTIN SORIA
}

Damos un informe breve de las fortificaciones de Extremadura. No agotamos el tema. Sabemos más detalles de tales defensas medievales, pero nos tenemos que reducir a una lista, monótona, necesariamente escueta, con algunas fechas de las fortificaciones de la provincia de Cáceres.

Existen atalayas extremeñas de Matamoros, en Alcántara; de Zapatería, en Brozas; del Cincho, en Brozas; de Arriba, en Trujillo; de Posia, en Trujillo; de Godoyes, en Cáceres; de Porcayos, en Cáceres; de Mayoralgo, en Cáceres; del Guijo, en Alcuéscar. Hay también atalayas en Acebo, en Santibáñez el Alto, en Puerto de Santa Cruz, en Torrequemada, en Casar de Cáceres, en Garganta la Olla, en Montehermoso.

Está la atalaya de Pelayo Vellido, construida por los árabes entre Coria y Galisteo.

Entre los castillos medievales extremeños citamos los siguientes: Acebuche, Alberquilla, Albalat, Alie, Alija (construido en 947 por Abdelrahmán III), Aliseda (atacado por el condestable portugués Nuño Alvarez Prerira en 1397), Almaraz (construido por Blanco Gómez de Almaraz en el siglo XIV), Almoharín (construido por los almorávides entre 1086 y 1145), Alpotreque (en el año 1264 se habla de este castillo y Fernando IV lo donó a Martín Gil de Sousa, conde de Barcelos, nieto y alférez mayor del rey trovador don Dionis de Portugal), Altamira, Torremenga (restos de murallas de la edificación construida por el tercer conde de Osorno), Arropez, Benavente de la Zarza (arrebatado por Fernando II de León a los árabes y reconquistado definitivamente por Alfonso IX en 1212, cedido a los Templarios por este monarca), Benfayán, Bernardo (conquistado por Fernando II de León en 1156), Boxe, Cabañas (en el camino de Trujillo a Guadalupe, siendo cabeza de señorío del mismo nombre de los Alvarez de Toledo, señores de Horcajo y Jarandilla, cuyo séptimo conde don Duarte. Fernando Alvarez de T'oledo y Portugal es también conde de Deleitosa, y Alcaudete, marqués de Jarandilla, conde de Oropesa y de Frechilla y Villarramiel), Sabeza de Esparragal (conquistado en enero de 1167 por Fernando II de León, donado a los Templarios, recuperado por los árabes en 1172 y adquirido en 1196 por los almohades, reconquistado en el siglo XIII por Alfonso IX y entregado por él a los freires del Perero), Cabezón (cedido por Fernando II, en 
1167, a los Templarios y situado entre Alconotar y Cañaveral), Cañamero, Casar del Conde (propiedad del conde Gonzalo Marañón en 1166 y 1170), Castellanos (castillo adjudicado por el rey de León a la familia Valverde, a raíz de la reconquista; Ruy González de Valverde fue uno

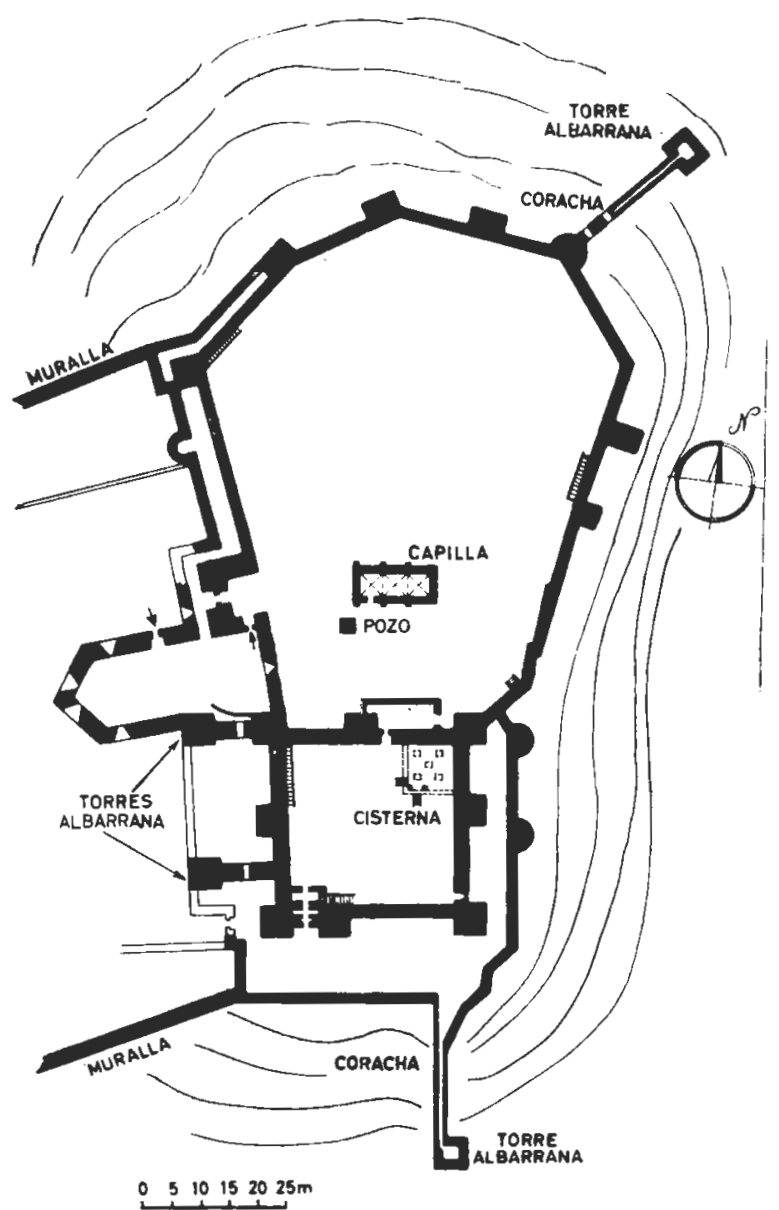

Castillo de Truillo, típico ejemplar árabe del siglo XI que ba conservado su planta original, aun cuando entre los siglos XIII y $X V$ se le añadiera la capilla y otros elementos. (Plano de F. Bordeje.)

de los capitanes que Fernando III de Castilla envió a su padre, Alfonso IX, para la conquista de las tierras comprendidas entre el río Tajo y el río Guadiana), Castiel Freyo (castillo mencionado en el libro de la Montería 
atribuido a Alfonso XI), Castil Dueñas (castillo perteneciente desde la reconquista a la familia Figueroa y luego al gran mariscal García González de Herrera).

Damos seguidamente una relación de castillejos de las siguientes localidades: Santibáñez, Santa Cruz de Paniagua, Granja de Granadilla, Arroyomolinos de Montánchez, Solana, Guadalupe, Garciaz, Robledollano, Malpartida de Plasencia, Casas de Don Gómez, Coria, Viandar de la Vera, Jarandilla (ruinas denominadas del Castillejo), Castillejo de Gutiérrez, Castillejo de la Orden, Castillejo de Arriba, Castillejo de la Buitrera, Castillejo de la Guadiloba, Castillejo del Salor.

Existían fortificaciones medievales en Castro (citado en el año 1296), en Ceclavín, en Coronada (construido en 1274 por los trujillanos), en

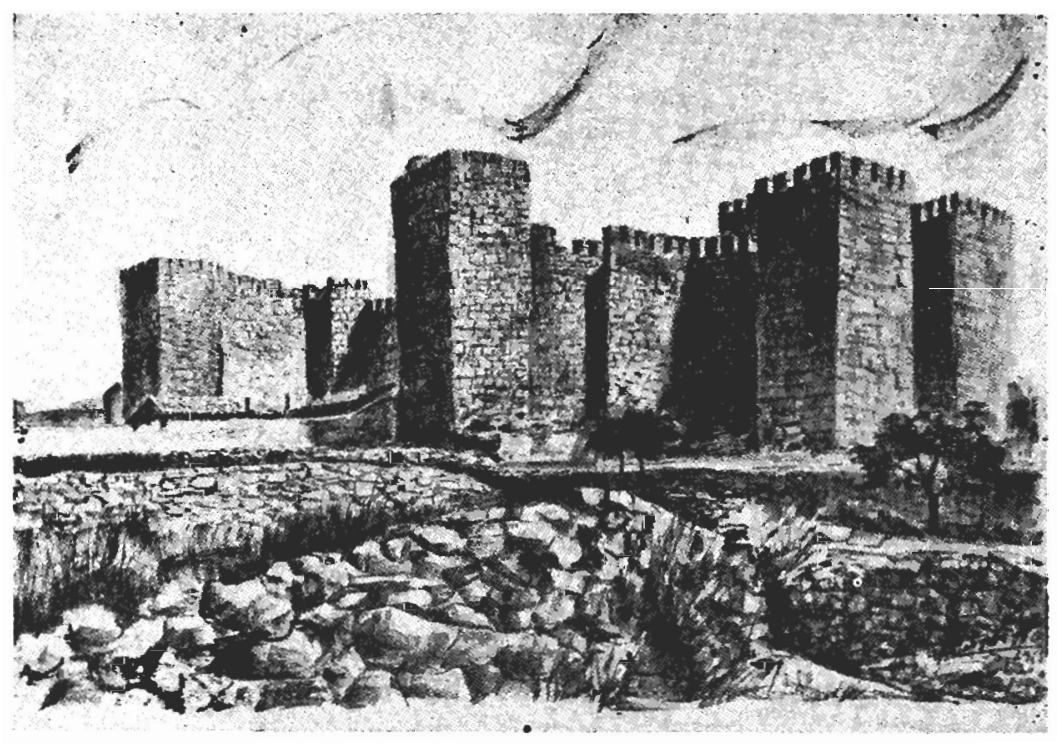

Castillo de Trujillo. (Dibujo de F. Castillo.)

Deleitosa, en Espejel (se menciona en 1290), en Fuentidueñas (construido por los Templarios en el siglo XII), en Malgarrida (donado por Enrique II a su camarero Lorenzo Fernández de Ulloa en el siglo xiv), en Miajadas (del siglo XIII), en Mignaza (junto al castillo de Mirabel), en Oliva de Plasencia (señorío de Fernán Pérez de Monroy, hermano del abad de Santander, señor de Valverde de la Vera, del siglo xIV, no muy lejos del 
arco cuádruple de Caparra y de sus ruinas romanas), en Palomera (donado por Alfonso IX el 31 de mayo de 1203 a la iglesia compostelana y en 1209 a la Orden de Santiago), en Peña de Fray Domingo (del siglo XII), en Peña Falcón (cedido por Pedro Fernández de Castro a la Orden de Alcántara en favor de su tío Fernando Díaz en 1187), en Piedras Albas (árabe conquistado por la Orden de Alcántara en el siglo XIII), en Ronda (conquistado por Alfonso VIII y entregado en 1195 a los freires de la Orden de Perero), en Santa Cruz (adquirido por los freires del Perero), Castillo de Xalama, Castillo de Xerit (aludido en el fuero dado por Alfonso IX en Coria), Castillo de Zufarola (árabe entre los ríos Tozo y Almonte.

Mencionamos una lista de torres defensivas con toda probabilidad de época medieval: Albarragena, en Valencia de Alcántara; Algodor, en Casillas de Coria; Bootello, en Alcántara; Cadalso, en Cadalso de la Sierra; Matacabras, en Alcántara; Oviedo, en Alcántara; Santo Domingo, en Granja de Granadilla; Cargas, en Miajadas; Velasco Muza, en Talavera; Ejido Nuevo, en Navalmoral de la Mata; Espigón, en Trujillo; Salgado, en Torrejoncillo; Marcos, en Madroñera. Y también citamos el arábigo castillo de Grimaldo, de la familia Sánchez de Grimaldo; castillo de Eljas, en Eljas, construido en el año 890.

Demos algunos datos breves sobre el castillo medieval de Montfrague. Los reyes de Castilla y León —Ordoño II, Ordoño III, Alfonso III, Fernando I- pudieron haber llegado $\mathrm{y}$ hasta conquistado esta fortaleza extremeña. Mientras Fernando II de León incorporaba a su corona los territorios de la sierra de Gata y los lugares enclavados en las riberas del Tajo, cuando llegó, en 1167, a la Cabeza del Esparragal después de haber conquistado Alcántara un guerrillero portugués llamado Giraldo Simpavor, se apoderó de diversas ciudades importantes, como Trujillo, Santa Cruz y Montfrague.

Al acudir el rey de León, Fernando, en 1169 en auxilio de los habitantes de Badajoz, para evitar que la comarca cayera en manos de Alonso Enríquez, apresó y derrotó a buen número de capitanes, prelados, condes y caballeros, entre ellos a Giraldo Simpavor. A este último le concedió la libertad Fernán Rodríguez, el castellano, entregando previamente Montánchez, Trujillo, Santa Cruz y Montfrague.

En tiempos de Alfonso VIII, en 1180, al fundar Plasencia, conquistó el castillo de Montfrague, que había sido cedido anteriormente, antes de ser conquistado por Abu Jacob, para los Caballeros de Montegaudio al conde don Rodrigo en 1172.

Un castillo medieval interesante es el de Montánchez. En 1167 se alude a la toma de Montánchez por el rey de Marruecos, y en 1184 volvió a ser conquistada por un hijo de Abu Jacob. En el año 1196 o 1197 los 
árabes se volvieron a apoderar de Montánchez y en 1210 Alfonso VIII lo conquista. Alfonso IX entrega al maestre de Santiago, Pedro González Mengo, el castillo de Montánchez. Una lista de comendadores que poseyeron el castillo de Montánchez: Pedro Iñíguez, en 1235; Gómez Fernández, en 1242; Pedro Veláiz, en 1242; Rodrigo Alcil, en 1250; Pedro Fernández Mata, en 1282; Vasco López, en 1330.

Casillo de Valencia de Alcántara.

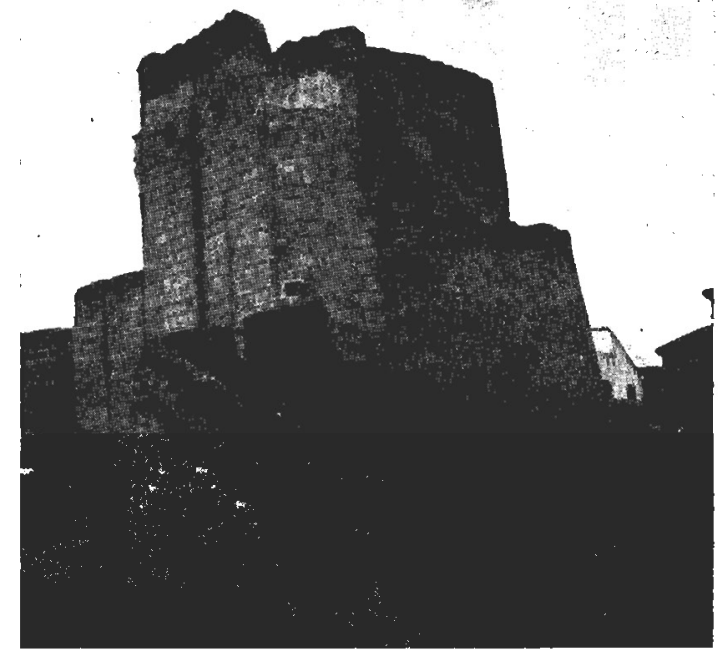

Posteriormente poseyeron el castillo de Montánchez los comendadores Pedro Ruiz de Sandoval, en 1383; Pedro Fernández, en 1384; Alonso de Portocarrero, en 1489; Alonso Enríquez, en 1494; Luis Portocarrero, en 1499.

El castillo de Peñafiel, de Zarza la Mayor, se llama también Racha Rachol. En 1166, el rey Fernando II de León cruzó la Carpetovetónica, liberó la sierra de Gata y también este castillo. Este castillo de Peñafiel fue encomienda de la Orden de Alcántara. Señalemos los nombres y fechas de los comendadores: frey Pedro Vázquez, en 1300; frey Hernando Arias, en 1335; frey Pedro Neyra, en 1343; frey Gonzalo Rodríguez, en 1355; frey García Gómez, en 1356; frey Martín Frade, en 1362; frey García Pérez, en 1365; frey Diego Fernández, en 1394; frey Juan de Soto, en 1416; frey Alonso de Peñaranda, en 1425; frey Pedro Altasangre, en 1431; frey Pedro de Salazar, en 1434; frey Diego Chamizo, en 1475; 
frey Francisco de Hinojosa (por usurpación), en 1477; frey Pedro Chamizo, en 1494. Fernando de Guzmán fue el primero que se tituló comendador de Peñafiel y la Zarza en 1496.

En los comienzos del siglo $\mathrm{x}$ fue construido el castillo de Portezuelo. Cerca del castillo hay algunos dólmenes y existe una gruta llamada Cueva de la Columna o Cancho de la Culera.

A mediados del siglo Ix, cuando estaba al frente de los destinos del pueblo en la provincia de Al-Kasser, Ibn Abu Danes, incluido en los territorios del Garb, el guerrillero de Mérida Aben Merwan, llamado el gallego, porque había concertado con el rey cristiano Alfonso III, actuaba por esta comarca. Sabemos que en 800 era jefe supremo de Coria y de Portezuelo cierto personaje que recibía el título de rey llamado Zeth.

Alfonso III, en 877, no logró conquistar la fortaleza de Portezuelo. En el mes de julio de 997, cuando Almanzor cruzó el puente de Alconeter y a través de Coria y del castillo de Portezuelo llegó hasta Santiago de Compostela.

En el año 1166, Fernando II de León conquistó el castillo de Salvaleón, llamado en latín Interannia, entre ríos; en 1221, el maestro de Alcántara, Arias Pérez, recibió el castillo de Salvaleón y sus tierras de Alfonso IX. Dos piedras romanas hablan de Interannia. Una que dice así: «Ianci - no. Celt - I. F. Inte - ranien - An. LXV. - Iancino, de sesenta y cinco años, natural de Interannia, hijo de Celta. - Esto fue encontrado en Salvaleón.» Existe también otra lápida sepulcral hallada en Mérida que dice sobre Salvaleón lo siguiente: «D. M. S. - Mativs Ferminv in - teraniensis. - Ann. LII. - H. S. E. S. T. T. L. - Memoria consagrada a los dioses muertos. Marco Acio Fermín, natural de Interannia, que murió de cincuenta y dos años, está aquí enterrado. Séate la tierra liviana.»

Presentamos la lista de comendadores de la Orden de Alcántara que rigieron en Salvaleón: frey Juan Pérez, en 1298; frey Men Rodríguez de Viedma, en 1300; frey Vasco Fernández, en 1335; frey Gonzalo Yáñez, en 1340; frey Fernando Alonso, en 1343; frey Rui Pérez de Busto, en 1355; frey Sancho Gutiérrez, en 1362; frey Alonso Fernández, en 1365; frey Fernando Díaz, en 1383; frey Fernán Fernández, en 1394; frcy Juan de San Juan, en 1416; frey Juan de Salazar, que ostentó ya el título de comendador de Salvaleón y de Eijas, en 1428.

El último maestre de Alcántara, Juan de Zúñiga, hijo de la célebre duquesa de Arévalo y Plasencia Leonor de Pimentel, al ser consultado por el concejo de Alcántara sobre quién o sobre quiénes debían facilitar a los respectivos maestres el vino que consumieran, falló la obligación sobre las villas de Salvaleón.

En el año 1166, Fernando II de León dio al maestre y a la Orden del Temple el castillo de Santibáñez, al arrebatárselo a los moros. Lo recu. 
peró en otra ocasión su hijo Alfonso IX, pues se había perdido. Se lu entregó a los freires del Pereró. En 1220, Pedro de Alviz, maestre del Temple, y sus caballeros acudieron a Alfonso para pedirle el castillo de Santibáñez, y en vez de ello, les entregó Alba de Liste. El primer comendador de Santibáñez de que tenemos noticia es frey Miguel Sánchez, familiar muy allegado a frey Garci Sánchez, que en 1217 era maestre de la Orden de Alcántara. Sucedió a frey Garci en el maestrazgo frey Arias Pérez.

Señalamos los comendadores de Santibáñez: frey Miguel Sánchez, en 1227; frey García Gómez, en 1294; frey Ruy González, en 1297; frey Suero Pérez, que después fue maestre, en 1316; frey Vasco Pérez, en 1318; frey Alvar Pérez, en 1335; frey Gonzalo Martínez, en 1340; frey Pedro Alonso, en 1340; frey Pedro Chamizo (hermano del maestre), en 1341; frey Suero Martínez, en 1345; frey Diego Pérez, en 1362; frey Rodrigo Alvarez, en 1364; frey Alonso Fernández, en 1367; frey Rodrigo Yáñez (hermano del maestre), en 1386; frey Luis González de Valdelomar, en 1394; frey Gonzalo Alvarez de Villagutiérrez, en 1403; frey Gonzalo Alvarez de Villasayas, en 1426; frey Alonso de Villasayas, en 1450; frey Pedro de Villasayas, en 1466; frey Luis de Villasayas, en 1494. Hemos querido dar algunos datos sobre fortalezas, castillos y torres de Extremadura medieval. Lista breve, rápida, corta, pero que pensamos ampliar con un extenso estudio. 